\title{
Classical Fluctuating Charge Theories: The Maximum Entropy Valence Bond Formalism and Relationships to Previous Models ${ }^{\dagger}$
}

\author{
Jorge Morales and Todd J. Martínez* \\ Department of Chemistry and The Beckman Institute, University of Illinois, Urbana, Illinois 61801
}

Received: October 17, 2000; In Final Form: January 26, 2001

\begin{abstract}
We explore the relationship between grand canonical (GC) ensemble and charge equilibration (CE), also known as electronegativity equalization, theories for describing charge flow in molecules. We introduce a new unifying approach to classical charge transfer theories based on valence bond (VB) theory and the maximum entropy (ME) method, which we call MEVB. We show how MEVB reduces to GC and CE theories with different choices for the definition of atomic partial charge. We believe that the MEVB approach provides a rigorous framework within which both improved classical models of charge transfer can be developed and a well-defined procedure for interfacing classical electrostatic models with quantum chemistry can be established.
\end{abstract}

\section{Introduction}

With few exceptions, ${ }^{1,2}$ quantum chemical calculations of electronic structure take the number of electrons in the molecule as a fixed parameter. This is usually the correct approach for a molecule in vacuo but can be called into question in the presence of a surrounding environment which may either donate or accept electrons. This point becomes important, for example, in the treatment of solvation phenomena, surface chemistry, and enzyme mechanisms. One traditional ab initio approach to modeling these problems is to apply quantum chemistry to a fragment of the system (the "active region," usually chosen to be a closed-shell molecule or collection of molecules). ${ }^{3-8}$ The idea is to exploit the locality of many chemical phenomena; the active region should obviously include any breaking or forming chemical bonds, but the success of empirical constructs like group additivity tables ${ }^{9}$ suggests that the detailed quantum mechanics of far-removed bonds will be unimportant. This fragment may then be immersed in a dielectric continuum ${ }^{10,11}$ or a field of point charges/dipoles ${ }^{12}$ in an effort to capture the electrostatic effects of the environment. Mixed quantum mechanical/molecular mechanical (QM/MM) approaches ${ }^{13-15}$ go further and include also environmental steric effects. ${ }^{16}$ When charge transfer to/from the environment is expected to be important, the conventional remedy is to increase the size of the active region. In many cases, this becomes impractical because of the computational cost of ab initio quantum chemistry. An attractive alternative would be to develop a "quantum chemistry for open systems", i.e., a grand canonical system-bath decomposition of the electronic structure problem. A very intriguing early effort along these lines, but in the context of the canonical ensemble, can be found in ref 17 .

The construction of models that allow for charge flow without explicit quantum chemical treatment of any of the constituent subsystems is closely related to the above issues-the only difference being that all of the subsystems are treated at a low level of detail. In particular, we are thinking of models based on atomic electronegativities and hardnesses. These charge equilibration (CE) methods rely on the electronegativity equal-

$\dagger$ Part of the special issue "William H. Miller Festschrift". ization (EE) principle of Sanderson ${ }^{18}$ and are variously known as chemical potential equalization (CPE), $\mathrm{CE}$, and $\mathrm{EE}$ methods. The conceptual framework for these methods is largely intuitive and draws heavily on seminal work by many authors. ${ }^{18-24}$ While highly approximate versions of such methods have existed for some time ${ }^{25,26}$ it is primarily within the past decade that serious numerical implementations and refinements have been carried out. ${ }^{27-30}$ Difficulties with these CE models have been noted, ${ }^{31}$ usually in the form of excess charge transfer and instability and often occurring when bonds are significantly stretched. Although some pragmatic solutions have been proposed, ${ }^{29,31}$ one can hope that a rigorous formulation of quantum chemistry for open systems will lead in a natural way to progress on this front also.

One of the practical reasons that we are interested in this problem is in the context of multiscale descriptions of intermolecular potential energy surfaces such as QM/MM methods. Two fundamental issues are of particular interest to us. First, the $\mathrm{QM} / \mathrm{MM}$ boundary in these approaches is typically fixed and particles are not allowed to cross from one region to the other. Yet there are many situations where it would be desirable to allow the boundaries to fluctuate or to allow electrons, atoms, and/or molecules to cross the boundary, for example, when chemistry is coupled to vectorial transport as in cytochrome $c$ oxidase. ${ }^{8}$ Second, there are intrinsically quantum mechanical terms in the interaction between classical and quantum subsystems, for which the most natural definition implicitly requires a wave function in both subsystems. One example is the shortrange repulsion arising from the Pauli exclusion principle. It is, of course, always possible to parametrize these interactions, but such a parametrization will depend on both subsystems and may not be transferable. We feel that an alternative approach of endowing both subsystems with a dual classical/quantum nature is more attractive. ${ }^{32}$ Other approaches that use such a dual representation in some regions have also been proposed, especially for QM/MM boundaries across covalent bonds. ${ }^{33-35}$

There is a practical problem in defining the subsystems to have both classical and quantum identities. If there is to be a compelling advantage over treating the entire system quantum mechanically, one must be able to move both up and down the hierarchy of representations. Going from a wave function-based 
description to a classical electrostatic representation is a simple matter of integration, but the reverse classical $\rightarrow$ quantum transformation is problematic because the map is obviously not information-preserving. In our previous attempts along these lines, ${ }^{32}$ we have used model wave functions that were stored (and could be perturbed if necessary). While this is reasonable for special cases, such as a quantum solute in a classical solvent, it will clearly become cumbersome when the classical region is not composed of many chemically identical molecules.

In this paper, we introduce one possible definition of this classical $\rightarrow$ quantum map that has some theoretical appeal. The main idea is to use the classical information, e.g., the atomic partial charges, as a set of constraints. This defines a reduced Hilbert space containing all wave functions consistent with the constraints. If the set of constraints is large enough, this reduced Hilbert space will contain only one wave function (within arbitrary phase and normalization factors). However, in the cases that interest us there will not be so many constraints as to fix the wave function uniquely. How then can we proceed? Perhaps the most natural option is to invoke an auxiliary equation; for example, one could apply a variational principle and find the wave function in the reduced space that minimizes the energy. From an information theoretic standpoint, this is just a compact way of specifying a full set of constraints. The clear disadvantage is that we are led immediately back to the quantum mechanical problem that we wish to avoid, albeit in a reduced Hilbert space. Without specifying any constraints beyond the original set, information theory gives us only one viable choice: apply a maximum entropy (ME) principle within the subspace consistent with the constraints. In practice, this means that all wave functions within the subspace must be considered with equal weight. This does not lead to a unique wave function, but rather to a unique prescription for any wave function-derived properties through an appropriate average, which is usually all that is required. This prescription is easily extended to cases with more constraints, for example atomic charges, dipoles, and polarizabilities.

Several comments must be made at the outset. First, we apply the ME principle in Hilbert space, i.e., directly to the wave function coefficients. This is in contrast to the more traditional approach that applies ME to the density matrix. However, it is easy to show that the two procedures are mathematically identical. Second, many chemical applications of ME treat the constraints as fixed parameters. For example, one might choose the mean value of an observable that has been measured. ${ }^{36}$ However, this is not at all necessary. ${ }^{37}$ One can instead view the variables as forming two sets: those which are deemed knowable and those which will not be known, either by choice or because they cannot be known in principle. The "knowable" variables then become the constraints and ME is applied to define an energy (or other property) for each possible realization of the constraints. Finally, one applies a physically motivated auxiliary equation, e.g., energy minimization, to determine the values of the constraints. Such a procedure combines physical and information theoretical considerations to find the best solution consistent with the restriction of predetermined maximal information content.

\section{Theory}

1. Charge Equilibration (CE) Approaches to Charge Transfer. The traditional heuristic "derivation" of the CE model starts from Iczokowsky and Margrave's analysis ${ }^{20}$ of the variation of the energy of an isolated atom as a function of its total charge. These authors suggested that one could fruitfully expand the energy of an atom in a Taylor series truncated at second order:

$$
\begin{aligned}
E_{\mathrm{A}}^{\mathrm{CE}}\left(q_{\mathrm{A}}\right) & =E_{\mathrm{A}}^{(0)}+\left.\frac{\partial E_{\mathrm{A}}}{\partial q_{\mathrm{A}}}\right|_{q_{\mathrm{A}}=0} q_{\mathrm{A}}+\left.\frac{1}{2} \frac{\partial^{2} E_{\mathrm{A}}}{\partial q_{\mathrm{A}}^{2}}\right|_{q_{\mathrm{A}}=0} q_{\mathrm{A}}^{2} \\
& =E_{\mathrm{A}}^{(0)}+\chi_{\mathrm{A}}^{0} q_{\mathrm{A}}+\eta_{\mathrm{A}}^{0} q_{\mathrm{A}}^{2}
\end{aligned}
$$

and further noticed that a 3-point finite difference approximation to the electronegativity yields the Mulliken ${ }^{24}$ definition:

$$
\chi_{\mathrm{A}}^{0}=\frac{\partial E_{\mathrm{A}}}{\partial q_{\mathrm{A}}}=-\mu_{\mathrm{A}}\left(q_{\mathrm{A}}=0\right) \approx \frac{1}{2}\left(I P_{\mathrm{A}}+E A_{\mathrm{A}}\right)
$$

where $\mathrm{IP}_{\mathrm{A}}$ and $\mathrm{EA}_{\mathrm{A}}$ are the ionization potential and electron affinity of the atom, and the equivalence of the electronegativity and the chemical potential $\mu$ has been noted. A similar treatment for the hardness ${ }^{38}$ gives

$$
\eta_{\mathrm{A}}^{0}=\frac{1}{2} \frac{\partial^{2} E_{\mathrm{A}}}{\partial q_{\mathrm{A}}^{2}} \approx \frac{1}{2}\left(\mathrm{IP}_{\mathrm{A}}-\mathrm{EA}_{\mathrm{A}}\right)
$$

Assuming additivity of the atomic energies, one can write the energy of a molecule containing $N$ atoms as

$$
\begin{aligned}
& E^{\mathrm{CE}}\left(q_{1}, \ldots q_{N}\right)=\sum_{\mathrm{A}}\left(E_{\mathrm{A}}^{(0)}+q_{\mathrm{A}} \chi_{\mathrm{A}}^{(0)}+q_{\mathrm{A}}^{2} \eta_{\mathrm{A}}^{(0)}\right)+ \\
& \sum_{\mathrm{A}>\mathrm{B}} q_{\mathrm{A}} q_{\mathrm{B}} J_{\mathrm{AB}}^{\mathrm{CE}}\left(R_{\mathrm{AB}}\right)
\end{aligned}
$$

where the Coulomb interactions between the partial charges has been incorporated. Early implementations used the simple asymptotic form $J_{\mathrm{AB}}^{\mathrm{CE}}\left(R_{\mathrm{AB}}\right) \rightarrow 1 / R_{\mathrm{AB}}$, but in their QEq method, Rappe and Goddard ${ }^{27}$ suggested a more sophisticated form that incorporates shielding effects that are important at typical bond distances (atomic units are used here and throughout this paper). Minimizing eq 2.4 under the constraint of fixed total charge leads to the optimal partial atomic charges and is equivalent to the application of Sanderson's electronegativity equalization principle. ${ }^{18}$ The resulting equations are a linear system that can be easily solved even for large molecules on modern computers. Given an adequate parametrization of the atomic electronegativities, hardnesses, and shielded Coulomb interaction, the $\mathrm{CE}$ equations can accurately predict atomic charges near the equilibrium geometry of a molecule. ${ }^{27,28}$ However, as will be shown in detail in later sections, the CE equations unfortunately predict physically incorrect charges at molecular dissociation in the gas phase. In fact, there are no satisfactory CE equations for reactive processes, posing a serious obstacle to the development of QM/MM methods that allow charge to flow in to and out of the "active region."

The work in this paper presents a more rigorous approach to the CE equations from a wave function viewpoint. Serious attempts to derive the $\mathrm{CE}$ equations have also been carried out previously from the viewpoint of density functional theory (DFT). ${ }^{39}$ These efforts trace back to the identification made by Parr et al. ${ }^{23}$ for the chemical potential as $\mu=\partial E[\rho] / \partial \rho$, where $E[\rho]$ is the energy functional in terms of the density $\rho$. Discussion of these earlier approaches is deferred until later in the paper.

2. Statistical Grand Canonical (GC) Treatment of Nonintegral Electronic Occupation. We now summarize the main features of the statistical description of an atom A capable of interchanging electrons with a surrounding thermal bath, as 
developed extensively by Parr and co-workers ${ }^{39,40}$ in the context of DFT, building on earlier work. ${ }^{41-43}$ The simplest version of the grand canonical (GC) ensemble description of an atom A considers only the pure ground states corresponding to the ionic species $\mathrm{A}^{0}, \mathrm{~A}^{+}$, and $\mathrm{A}^{-}$. Although there does exist a more complex version of this theory ${ }^{39}$ that takes into account species of any number of electrons and their excited states, it leads to very similar results in the limit of most practical interest, where $\beta=1 / k T \rightarrow \infty$. Therefore, we confine our attention to the simplest version for the remainder of this manuscript.

The $\mathrm{GC}$ partition function $Z(\beta, \mu)$ of the atom $\mathrm{A}$ is given as

$$
Z(\beta, \mu)=\sum_{i} g_{i} \mathrm{e}^{-\beta\left(E_{i}-\mu N_{i}\right)}
$$

where $i$ labels the charge and electronic states included in the ensemble, $N_{i}$ labels the number of electrons in the $i$ th state, $E_{i}$ is the state energy, $\mu$ is the chemical potential, and $g_{i}$ is the degeneracy of the $i$ th state. Restricting to the case of neutral and singly charged ground electronic states, eq 2.5 can be rewritten as

$$
\begin{aligned}
Z(\beta, \mu)=\exp \left[-\beta\left(E_{\mathrm{A}^{0}}-\mu N_{\mathrm{A}^{0}}\right)\right] \\
\left\{g_{\mathrm{A}^{0}}+g_{\mathrm{A}^{+}} \exp \left[-\beta\left(\mathrm{IP}_{\mathrm{A}}+\mu\right)\right]+\right. \\
\left.g_{\mathrm{A}^{-}} \exp \left[+\beta\left(\mathrm{EA}_{\mathrm{A}}+\mu\right)\right]\right\}
\end{aligned}
$$

For convenience in what follows, we now specialize to the case of a doublet neutral ground state and singlet charged states, where $g_{\mathrm{A}^{0}}=2$ and $g_{\mathrm{A}^{+}}=g_{\mathrm{A}^{-}}=1$. The average number of electrons $\left\langle N_{\mathrm{A}}\right\rangle$ and the average energy $\left\langle E_{\mathrm{A}}\right\rangle$ are then

$$
\begin{aligned}
\left\langle N_{\mathrm{A}}\right\rangle= & \frac{1}{\beta}\left(\frac{\partial \ln Z}{\partial \mu}\right)_{\beta} \\
= & N_{\mathrm{A}^{0}}+ \\
& \frac{\exp \left[\beta\left(\mathrm{EA}_{\mathrm{A}}+\mu\right)\right]-\exp \left[-\beta\left(\mathrm{IP}_{\mathrm{A}}+\mu\right)\right]}{\left\{2+\exp \left[-\beta\left(\mathrm{IP}_{\mathrm{A}}+\mu\right)\right]+\exp \left[\beta\left(\mathrm{EA}_{\mathrm{A}}+\mu\right)\right]\right\}}
\end{aligned}
$$

and

$$
\begin{aligned}
\left\langle E_{\mathrm{A}}\right\rangle & =-\left(\frac{\partial \ln Z}{\partial \beta}\right)_{\mu} \\
& =E_{\mathrm{A}^{0}}- \\
& \frac{E A_{\mathrm{A}} \exp \left[\beta\left(\mathrm{EA}_{\mathrm{A}}+\mu\right)\right]-\mathrm{IP}_{\mathrm{A}} \exp \left[-\beta\left(\mathrm{IP}_{\mathrm{A}}+\mu\right)\right]}{\left\{2+\exp \left[-\beta\left(\mathrm{IP}_{\mathrm{A}}+\mu\right)\right]+\exp \left[\beta\left(\mathrm{EA}_{\mathrm{A}}+\mu\right)\right]\right\}}
\end{aligned}
$$

and the atomic charge can be defined as

$$
\begin{aligned}
q_{\mathrm{A}} & =\left(N_{\mathrm{A}^{0}}-\left\langle N_{\mathrm{A}}\right\rangle\right) \\
& =\frac{-\exp \left[\beta\left(\mathrm{EA}_{\mathrm{A}}+\mu\right)\right]+\exp \left[-\beta\left(\mathrm{IP}_{\mathrm{A}}+\mu\right)\right]}{\left\{2+\exp \left[-\beta\left(\mathrm{IP}_{\mathrm{A}}+\mu\right)\right]+\exp \left[\beta\left(\mathrm{EA}_{\mathrm{A}}+\mu\right)\right]\right\}}
\end{aligned}
$$

One can easily show that the chemical potential, $\mu$, may be regarded as a function of the atomic charge and inverse temperature, i.e., $\mu=\mu\left(q_{\mathrm{A}}, \beta\right)$. For the case where $q_{\mathrm{A}}=0$, one finds $\mu_{0}=\mu\left(q_{\mathrm{A}}=0, \beta\right)=-\left(I P_{\mathrm{A}}+E A_{\mathrm{A}}\right) / 2$, which is the wellknown temperature-independent result for the chemical potential. For other values of the atomic charge, $\mu$ will have an explicit temperature dependence. Restricting our attention to large but finite reciprocal temperatures, we define $\mathrm{P}\left(q_{\mathrm{A}}, \beta\right)=\mu\left(q_{\mathrm{A}}, \beta\right)-$ $\mu_{0}$. An explicit expression for this function has been obtained previously, ${ }^{39,40}$ but we only require knowledge of the function's sign at high values of $\beta$, which can be deduced from the chemically reasonable assumption that $\mathrm{IP}_{\mathrm{A}}-\mathrm{EA}_{\mathrm{A}}>0$ :

$$
\mathrm{P}\left(q_{\mathrm{A}}, \beta\right)=\mu\left(q_{\mathrm{A}}, \beta\right)-\mu_{0}= \begin{cases}0 & \text { if } q_{\mathrm{A}}=0 \\ <0 & \text { if } q_{\mathrm{A}}>0 \\ >0 & \text { if } q_{\mathrm{A}}<0\end{cases}
$$

From eqs 2.8 and 2.9 and the definition of $\mathrm{P}\left(q_{\mathrm{A}}, \beta\right)$,

$$
\begin{array}{r}
E_{\mathrm{A}}^{\mathrm{GC}}=E_{\mathrm{A}^{0}}+q_{\mathrm{A}} \mathrm{EA}_{\mathrm{A}} \frac{1}{2}\left\{1+\operatorname{coth}\left[\beta \mathrm{P}\left(q_{\mathrm{A}}, \beta\right)\right]\right\}+ \\
q_{\mathrm{A}} \mathrm{IP}_{\mathrm{A}} \frac{1}{2}\left\{1-\operatorname{coth}\left[\beta \mathrm{P}\left(q_{\mathrm{A}}, \beta\right)\right]\right\}
\end{array}
$$

To make contact with CE theories, it is helpful to rewrite this explicitly as a weighted sum:

$$
E_{\mathrm{A}}^{\mathrm{GC}}=w_{\mathrm{A}^{0}}^{\mathrm{GC}} E_{\mathrm{A}^{0}}+w_{\mathrm{A}^{+}}^{\mathrm{GC}} E_{\mathrm{A}^{0}}+w_{\mathrm{A}^{-}}^{\mathrm{GC}} E_{\mathrm{A}^{0}}
$$

where

$$
\begin{aligned}
& w_{\mathrm{A}^{0}}^{\mathrm{GC}}=1+q_{\mathrm{A}} \operatorname{coth}\left[\beta \mathrm{P}\left(q_{\mathrm{A}}, \beta\right)\right] \\
& w_{\mathrm{A}^{+}}^{\mathrm{GC}}=\frac{q_{\mathrm{A}}}{2}\left\{1-\operatorname{coth}\left[\beta \mathrm{P}\left(q_{\mathrm{A}}, \beta\right)\right]\right\} \\
& w_{\mathrm{A}^{-}}^{\mathrm{GC}}=-\frac{q_{\mathrm{A}}}{2}\left\{1+\operatorname{coth}\left[\beta \mathrm{P}\left(q_{\mathrm{A}}, \beta\right)\right]\right\}
\end{aligned}
$$

In the limit where $\beta \rightarrow \infty(T \rightarrow 0)$,

$$
\begin{aligned}
& E_{\mathrm{A}}^{\mathrm{GC} \beta \rightarrow \infty}\left(q_{\mathrm{A}}\right)=E_{\mathrm{A}^{0}}+\mathrm{EA}_{\mathrm{A}} q_{\mathrm{A}}\left[1-\Theta\left(q_{\mathrm{A}}\right)\right]+\mathrm{IP}_{\mathrm{A}} q_{\mathrm{A}} \Theta\left(q_{\mathrm{A}}\right) \\
& =\left\{\begin{array}{llr}
E_{\mathrm{A}^{0}} & \text { if } & q_{\mathrm{A}}=0 \\
E_{\mathrm{A}^{0}}+\mathrm{IP}_{\mathrm{A}} q_{\mathrm{A}} & \text { if } & 0<q_{\mathrm{A}}<1 \\
E_{\mathrm{A}^{0}}+\mathrm{EA}_{\mathrm{A}} q_{\mathrm{A}} & \text { if } & -1<q_{\mathrm{A}}<0
\end{array}\right.
\end{aligned}
$$

and

$$
\begin{aligned}
& w_{\mathrm{A}^{0}}^{\mathrm{GC} \beta \rightarrow \infty}=1+q_{\mathrm{A}}-2 q_{\mathrm{A}} \Theta\left(q_{\mathrm{A}}\right) \\
& w_{\mathrm{A}^{+}}^{\mathrm{GC} \beta \rightarrow \infty}=q_{\mathrm{A}} \Theta\left(q_{\mathrm{A}}\right) \\
& w_{\mathrm{A}^{-}}^{\mathrm{GC} \beta \rightarrow \infty}=q_{\mathrm{A}}\left[\Theta\left(q_{\mathrm{A}}\right)-1\right]
\end{aligned}
$$

where $\Theta$ is the Heaviside step function. ${ }^{44}$ Since $^{39} \mu\left(q_{\mathrm{A}}, \beta \rightarrow \infty\right)$ $=-\partial E_{\mathrm{A}}^{\mathrm{GC} \beta \rightarrow \infty} / \partial q_{\mathrm{A}}$, one also finds

$$
\begin{aligned}
\mu\left(q_{\mathrm{A}}, \beta \rightarrow \infty\right)= & \operatorname{EA}_{\mathrm{A}}\left[\Theta\left(q_{\mathrm{A}}\right)-1\right]+\mathrm{EA}_{\mathrm{A}} q_{\mathrm{A}} \delta\left(q_{\mathrm{A}}\right)- \\
& \operatorname{IP}_{\mathrm{A}} \Theta\left(q_{\mathrm{A}}\right)-\mathrm{IP}_{\mathrm{A}} q_{\mathrm{A}} \delta\left(q_{\mathrm{A}}\right) \\
= & \begin{cases}-\frac{\left(\mathrm{EA}_{\mathrm{A}}+\mathrm{IP}_{\mathrm{A}}\right)}{2} & \text { if } \quad q_{\mathrm{A}}=0 \\
-\mathrm{IP}_{\mathrm{A}} & \text { if } 0<q_{\mathrm{A}}<1 \\
-\mathrm{EA}_{\mathrm{A}} & \text { if }-1 q_{\mathrm{A}}<0\end{cases}
\end{aligned}
$$

where $\delta\left(q_{\mathrm{A}}\right)$ is the Dirac delta function. ${ }^{44}$ The last expression provides a statistical basis for the Mulliken ${ }^{24}$ definition of electronegativity. Notice the piecewise linearity of $E_{\mathrm{A}}^{\mathrm{GC} \beta \rightarrow \infty}$ and the resulting derivative discontinuities in $\mu\left(q_{\mathrm{A}}, \beta \rightarrow \infty\right)$, which have been pointed out previously. ${ }^{40} \mathrm{~A}$ similar treatment of the hardness $\eta_{\mathrm{A}}^{0}$ also gives the expected result: ${ }^{39}$ 


$$
\eta_{\mathrm{A}}^{0}\left(q_{\mathrm{A}}=0, \beta \rightarrow \infty\right)=\frac{1}{2}\left[\frac{\partial^{2} E_{\mathrm{A}}^{\mathrm{GC} \beta \rightarrow \infty}\left(q_{\mathrm{A}}=0\right)}{\partial q_{\mathrm{A}}^{2}}\right]=\frac{1}{2}\left(\mathrm{IP}_{\mathrm{A}}-\mathrm{EA}_{\mathrm{A}}\right)
$$

3. Relation between $\mathrm{CE}$ and GC Treatments. Unlike $E_{\mathrm{A}}^{\mathrm{GC} \beta \rightarrow \infty}\left(q_{\mathrm{A}}\right), E_{\mathrm{A}}^{\mathrm{CE}}\left(q_{\mathrm{A}}\right)$ is a smooth function of $q_{\mathrm{A}}$, continuous up to its second derivative. Thus, $E_{\mathrm{A}}^{\mathrm{CE}}\left(q_{\mathrm{A}}\right)$ is obviously not equal to $E_{\mathrm{A}}^{\mathrm{GC} \beta \rightarrow \infty}\left(q_{\mathrm{A}}\right)$, but both predict correctly the electronegativity, $\chi_{\mathrm{A}}^{0}$, and hardness, $\eta_{\mathrm{A}}^{0}$, for the neutral atom. The natural question at this point is whether $E_{\mathrm{A}}^{\mathrm{CE}}\left(q_{\mathrm{A}}\right)$ is somehow related to $E_{\mathrm{A}}^{\mathrm{GC} \beta \rightarrow \infty}\left(q_{\mathrm{A}}\right)$ as a statistical average. Simple rearrangement of eq 2.4 :

$$
\begin{aligned}
E_{\mathrm{A}}^{\mathrm{CE}}\left(q_{\mathrm{A}}\right)=\left(1-q_{\mathrm{A}}^{2}\right) E_{\mathrm{A}^{0}}+ & \frac{1}{2}\left(q_{\mathrm{A}}^{2}+q_{\mathrm{A}}\right) E_{\mathrm{A}^{+}}+ \\
& \frac{1}{2}\left(q_{\mathrm{A}}^{2}-q_{\mathrm{A}}\right) E_{\mathrm{A}^{-}} \\
= & w_{0}^{\mathrm{CE}} E_{\mathrm{A}^{0}}+w_{+}^{\mathrm{CE}} E_{\mathrm{A}^{+}}+w_{-}^{\mathrm{CE}} E_{\mathrm{A}^{-}} \quad\left(-1 \leq q_{\mathrm{A}} \leq 1\right)
\end{aligned}
$$

gives the outward appearance of such an average. However, in order that $E_{\mathrm{A}}^{\mathrm{CE}}\left(q_{\mathrm{A}}\right)$ be a true statistical mechanics average, ${ }^{45}$ the weights must be positive semidefinite, and sum to unity. Although the second condition is satisfied, the first is not, because $w_{+}^{\mathrm{CE}}$ and $w_{-}^{\mathrm{CE}}$ are negative when $q_{\mathrm{A}} \in(-1,0)$ and $q_{\mathrm{A}} \in$ $(0,+1)$, respectively. One must conclude that the weights $w_{0}^{\mathrm{CE}}$, $w_{+}^{\mathrm{CE}}$, and $w_{-}^{\mathrm{CE}}$ define a spurious ensemble. Nevertheless, it is easily verified that $q_{\mathrm{A}}$ is the average charge $\left\langle q_{\mathrm{A}}\right\rangle$ within this ensemble, so that the average energy and average charge are consistent.

Despite this initial result, it is interesting to ask whether there is some less obvious way of relating the GC and $\mathrm{CE}$ theories. Somewhat surprisingly, this can be achieved by imposing only one constraint in $E_{\mathrm{A}}^{\mathrm{CE}}$, eq 2.11:

$$
\begin{aligned}
\operatorname{coth}\left[\beta \mathrm{P}\left(q_{\mathrm{A}}, \beta\right)\right] & =-q_{\mathrm{A}} \\
\Longrightarrow \beta \mathrm{P}\left(q_{\mathrm{A}}, \beta\right) & =\operatorname{coth}^{-1}\left(-q_{\mathrm{A}}\right) \\
= & \frac{1}{2} \ln \left(\frac{q_{\mathrm{A}}-1}{q_{\mathrm{A}}+1}\right) \quad\left(-1 \leq q_{\mathrm{A}} \leq+1\right)
\end{aligned}
$$

This constraint maps the weights and energies in the GC approach onto those predicted by charge equilibration. However, if $\beta \mathrm{P}\left(q_{\mathrm{A}}, \beta\right)$ is required to be real, this constraint is only realizable for $q_{\mathrm{A}}= \pm 1$. The remaining values of $q_{\mathrm{A}} \in(-1,+1)$ can only be realized by analytic continuation of the argument $\beta \mathrm{P}\left(q_{\mathrm{A}}, \beta\right)=\beta\left[\mu\left(q_{\mathrm{A}}, \beta\right)-\mu_{0}\right]$ :

$$
\beta \mathrm{P}\left(q_{\mathrm{A}}, \beta\right)=\frac{1}{2} \ln \left(\frac{1-q_{\mathrm{A}}}{q_{\mathrm{A}}+1}\right)+\frac{1}{2} \pi i \quad\left(-1<q_{\mathrm{A}}<+1\right)
$$

Since complex values of the function $\mathrm{P}\left(q_{\mathrm{A}}, \beta\right)$ have no clear meaning, it is most natural to effect this equivalence by choosing complex $\beta$. In this way, one can understand the origin of $\mathrm{CE}$ theories as complex temperature subductions of a rigorous statistical theory. The physically relevant effect of the analytical continuation is to smooth out the piecewise-linear behavior of $E_{\mathrm{A}}^{\mathrm{GC}}\left(q_{\mathrm{A}}\right)$. A similar smoothing of the derivative discontinuities in $E_{\mathrm{A}}^{\mathrm{GC}}\left(q_{\mathrm{A}}\right)$ can also be obtained at very small values of $\beta$ within the real domain. ${ }^{39}$ However, the curved shape of $E_{\mathrm{A}}^{\mathrm{GC}}\left(q_{\mathrm{A}}\right)$ generated by such physical heating is not that of
$E_{\mathrm{A}}^{\mathrm{CE}}\left(q_{\mathrm{A}}\right)$. We postpone further investigation of the role of complex temperature in charge transfer theories to future work. What is important for the present discussion is that there is no simple way to generate the $E_{\mathrm{A}}^{\mathrm{CE}}$ expression by conventional statistical mechanics methods without ad hoc constraints.

4. Analytical Aspects of the GC and CE Theories. It is very instructive to study in some detail the analytical behavior of both $E_{\mathrm{A}}^{\mathrm{GC} \beta \rightarrow \infty}\left(q_{\mathrm{A}}\right)$ and $E_{\mathrm{A}}^{\mathrm{CE}}\left(q_{\mathrm{A}}\right)$ for the case of a single atom. In the absence of specific charge-dependent system-bath interaction, ${ }^{46} E_{\mathrm{A}}^{\mathrm{GC} \beta \rightarrow \infty}\left(q_{\mathrm{A}}\right)$ is monotonically nonincreasing with decreasing $q_{A}$, i.e., as electrons are added to the atom. Given the restriction to the interval $q_{\mathrm{A}} \in[-1,1]$, the absolute minimum is therefore obtained for $q_{\mathrm{A} \text {,min }}=-1$. Since the derivative $\partial E_{\mathrm{A}}^{\mathrm{GC} \beta \rightarrow \infty}\left(q_{\mathrm{A}}\right) / \partial q_{\mathrm{A}}$ does not exist for integer $q_{\mathrm{A}}$, this minimum does not satisfy the condition $\partial E_{\mathrm{A}}^{\mathrm{GC} \beta \rightarrow \infty}\left(q_{\mathrm{A}, \min }^{\mathrm{GC} \beta \rightarrow \infty}\right) / \partial q_{\mathrm{A}}=0$. Extension of the GC theory to include more charge states does not affect this qualitative conclusion; the energy remains piecewise-linear with derivative discontinuities at integer charges, and the minimum energy is always obtained for the largest negative charge allowed. ${ }^{39}$ The situation is quite different for $E_{\mathrm{A}}^{\mathrm{CE}}\left(q_{\mathrm{A}}\right)$. Being a second-order interpolating polynomial, this function will exhibit a true minimum where $\partial E_{\mathrm{A}}^{\mathrm{CE}}\left(q_{\mathrm{A}}\right) / \partial q_{\mathrm{A}}=0$ for $q_{\mathrm{A}, \min }^{\mathrm{CE}}=-\left(\chi_{\mathrm{A}}^{0} / 2 \eta_{\mathrm{A}}^{0}\right)$. Since $\chi_{\mathrm{A}}^{0}>0$ and $\eta_{\mathrm{A}}^{0}>0$ (convexity), $q_{\mathrm{A}, \min }^{\mathrm{CE}}<0$, although this minimum need not lie in the interval $[-1,1]$. The minimum in $E_{\mathrm{A}}^{\mathrm{CE}}\left(q_{\mathrm{A}}\right)$ is an artifact of the interpolation procedure and has no obvious physical meaning. The physically incorrect consequences of this behavior are apparent when one considers a diatomic molecule; vide infra. The inclusion of additional charged species of the atom A to construct a higher-order interpolating polynomial does not solve the problem; it renders complicated expressions for the electronegativity and hardness that are difficult to interpret, and the resulting polynomial will be plagued with several unphysical maxima and minima.

Now we analyze the behavior of both $E_{\mathrm{A}}^{\mathrm{GC} \beta \rightarrow \infty}\left(q_{\mathrm{A}}\right)$ and $E_{\mathrm{A}}^{\mathrm{CE}}\left(q_{\mathrm{A}}\right)$ in the context of a dissociating molecule in the gas phase. Defining analogous energy expressions for an atom B, the total energy of a molecule $\mathrm{AB}$ at infinite separation is given by

$$
\begin{aligned}
& E_{\mathrm{AB}}^{\mathrm{GC} \beta \rightarrow \infty}\left(q_{\mathrm{A}}, q_{\mathrm{B}}, R_{\mathrm{AB}}=\infty\right)= \\
& E_{\mathrm{A}}^{\mathrm{GC} \beta \rightarrow \infty}\left(q_{\mathrm{A}}\right)+E_{\mathrm{B}}^{\mathrm{GC} \beta \rightarrow \infty}\left(q_{\mathrm{B}}\right) \\
& =\left(E_{\mathrm{A}^{0}}+E_{\mathrm{B} 0}\right)[1+q-2 q \Theta(q)]+ \\
& \left(E_{\mathrm{A}^{-}}+E_{\mathrm{B}^{+}}\right) q[\Theta(q)-1]+ \\
& \left(E_{\mathrm{A}^{+}}+E_{\mathrm{B}^{-}}\right) q \Theta(q)
\end{aligned}
$$

and

$$
\begin{aligned}
E_{\mathrm{AB}}^{\mathrm{CE}}\left(q_{\mathrm{A}}, q_{\mathrm{B}}, R_{\mathrm{AB}}=\infty\right)= & E_{\mathrm{A}}^{\mathrm{CE}}\left(q_{\mathrm{A}}\right)+E_{\mathrm{B}}^{\mathrm{CE}}\left(q_{\mathrm{B}}\right) \\
= & E_{\mathrm{A}^{0}}+E_{\mathrm{B}^{0}}+\left(\chi_{\mathrm{A}}^{0}-\chi_{\mathrm{B}}^{0}\right) q+ \\
& \left(\eta_{\mathrm{A}}^{0}+\eta_{\mathrm{B}}^{0}\right) q^{2}
\end{aligned}
$$

where $R_{\mathrm{AB}}$ is the bond distance and charge neutrality has been enforced by introducing $q=q_{\mathrm{A}}=-q_{\mathrm{B}} \Longrightarrow q_{\mathrm{A}}+q_{\mathrm{B}}=0$. Since both $E_{\mathrm{A}}^{\mathrm{GC} \beta \rightarrow \infty}\left(q_{\mathrm{A}}\right)$ and $E_{\mathrm{B}}^{\mathrm{GC} \beta \rightarrow \infty}\left(q_{\mathrm{B}}\right)$ are piecewise-linear with derivative discontinuities at integer charges, their sum $E_{\mathrm{AB}}^{\mathrm{GC} \beta \rightarrow \infty}\left(q, R_{\mathrm{AB}}=\infty\right)$ is also piecewise-linear with the same derivative discontinuities. In any chemically realistic case, $E_{\mathrm{A}^{0}}+E_{\mathrm{B}^{0}}$ is lower in energy than both $E_{\mathrm{A}^{+}}+E_{\mathrm{B}^{-}}$and $E_{\mathrm{A}^{-}}+$ $E_{\mathrm{B}}{ }^{+}$, ensuring that the absolute minimum is obtained for 


$$
q_{\min }^{\mathrm{GC} \beta \rightarrow \infty}=0
$$

i.e., dissociation in the gas-phase results in neutral atoms. As with $E_{\mathrm{A}}^{\mathrm{GC} \beta \rightarrow \infty}\left(q_{\mathrm{A}}\right)$ and $E_{\mathrm{B}}^{\mathrm{GC} \beta \rightarrow \infty}\left(q_{\mathrm{B}}\right)$, the minimum of $E_{\mathrm{AB}}^{\mathrm{GC} \beta \rightarrow \infty}\left(q, R_{\mathrm{AB}}=\infty\right)$ does not satisfy the condition $\partial E_{\mathrm{AB}}^{\mathrm{GC} \beta \rightarrow \infty}\left(q_{\min }^{\mathrm{GC} \beta \rightarrow \infty}\right) / \partial q=0$ because the derivative does not exist for integer charges. The optimization of $E_{\mathrm{AB}}^{\mathrm{GC} \beta \rightarrow \infty}(q, R=\infty)$ with respect to $q$ is therefore a case of linear programming. Furthermore, at the minimum, it does not hold that both atoms are at equilibrium among themselves, $\mu_{\mathrm{A}} \neq \mu_{B}$. This behavior is shown schematically in the left panel of Figure 1 for the specific case of LiF.

In contrast, the minimum of $E_{\mathrm{AB}}^{\mathrm{CE}}(q, R=\infty)$ does correspond to a point where $\partial E_{\mathrm{AB}}^{\mathrm{CE}}(q) / \partial q=0$, and

$$
q_{\mathrm{min}}^{\mathrm{CE}}=\frac{\left(\chi_{\mathrm{B}}^{0}-\chi_{\mathrm{A}}^{0}\right)}{2\left(\eta_{\mathrm{A}}^{0}+\eta_{\mathrm{B}}^{0}\right)}
$$

Since it always holds that $\eta_{\mathrm{A}}^{0}>0$ and $\eta_{\mathrm{B}}^{0}>0$ (convexity), the direction of the charge transfer is solely determined by the electronegativity difference $\chi_{\mathrm{B}}^{0}-\chi_{\mathrm{A}}^{0}$. Except when $\chi_{\mathrm{B}}^{0}-\chi_{\mathrm{A}}^{0}=$ 0 , this minimum will not lie at the physically correct point of $q_{\mathrm{min}}^{\mathrm{CE}}=0$, incorrectly predicting partially charged atoms at dissociation. This behavior is illustrated in the right panel of Figure 1 for the specific case of LiF. However, in the CE model, atoms $\mathrm{A}$ and $\mathrm{B}$ are at equilibrium, $\mu_{\mathrm{A}}=\mu_{\mathrm{B}}$. The root of the unphysical charge transfer can be traced back either to this enforced equilibrium or to the lack of derivative discontinuities at integer charges in the $\mathrm{CE}$ model. We do not find any compelling argument to insist on either of these alternative viewpoints.

Within the GC model, the infinitely remote atoms A and B are not at equilibrium but rather totally disconnected. Therefore, they will not exchange charge. In contrast, the same remote atoms described within the $\mathrm{CE}$ model are forced into an unphysical equilibrium, predicting incorrect charge transfer. There have been attempts ${ }^{47}$ to predict dissociation into neutral fragments while retaining the assumption of equilibrium between the fragments at infinite separation. However, this has been accomplished in an ad hoc manner, essentially by neglecting the electronegativity difference in the numerator of eq 2.24. Although such an approach may be worthy of more detailed consideration, we make no such attempt here.

Regardless of the appropriateness of $E_{\mathrm{AB}}^{\mathrm{GC} \beta \rightarrow \infty}\left(q, R_{\mathrm{AB}}\right)$ in the dissociated limit, one expects on physical grounds that as $R_{\mathrm{AB}}$ becomes finite, charge transfer may occur and equilibrium conditions will be obeyed. Thus, one expects the piecewiselinear form of $E_{\mathrm{AB}}^{\mathrm{GC} \beta \rightarrow \infty}\left(q, R_{\mathrm{AB}}\right)$ should become smoothed as the chemical bond is formed. Indeed, it has been speculated in the literature that the increasing interaction between the atoms when a molecule is made would bring the shape of $E_{\mathrm{AB}}^{\mathrm{GC} \beta \rightarrow \infty}(q, R=\infty)$ into one similar to that of $E_{\mathrm{AB}}^{\mathrm{CE}}(q) .{ }^{39}$ Although a statistical theory having these features would be highly desirable, it is not known at present. We take a completely different, wave function-based, approach in the remainder of this paper, but we note in passing that the complex temperature isomorphism presented above may provide a solution more in keeping with the spirit of the statistical approach.

5. Valence Bond (VB) Wave Function Approach and Its Relation with GC and CE Theories. So far, the discussion about a classical charge-transfer theory has been restricted to its traditional realm of statistical mechanics, with emphasis on

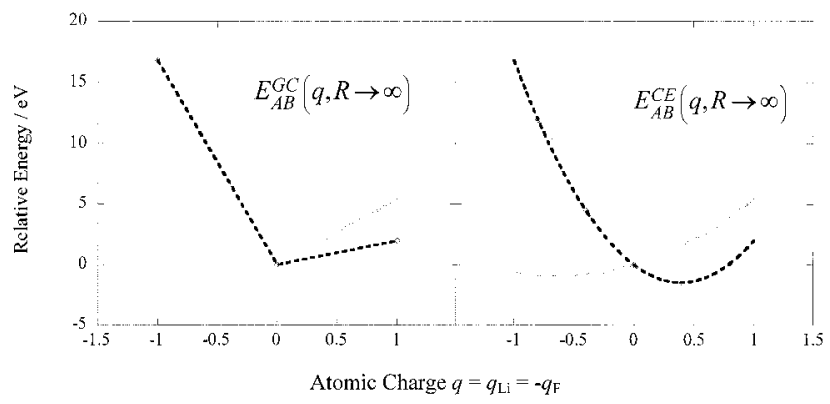

Figure 1. Dependence of energy on charge transfer in LiF at infinite separation for the GC and CE models. Solid lines are for the individual atoms, while the dashed lines denote the $\mathrm{LiF}$ molecule. Notice the piecewise-linear form of the energy in the GC model compared to the parabolic form in the CE model. The absolute minimum in the GC model is at $q=0$, as expected on physical grounds. As discussed in the text, there is residual charge transfer at infinite separation for the $\mathrm{CE}$ model, indicated by the fact that the energy is minimized for nonzero $q$.

the connection, or lack thereof, between GC and CE theories. In this section, we attempt to derive GC and $\mathrm{CE}$ theories directly from wave function-based quantum mechanics. The point is to identify exactly what assumptions would give rise to the GC and $\mathrm{CE}$ theories starting from first-principles quantum mechanics. While there have been some previous attempts in this direction, ${ }^{48}$ our approach is quite different. Furthermore, we believe our approach is the first to unify the GC and CE theories, both of which appear as special cases of a single theory in the following.

Our starting point is the simplest wave function capable of describing charge transfer in the system. First, we limit consideration to a diatomic molecule, not out of necessity but rather for clarity of exposition. For this case of a diatomic molecule $\mathrm{AB}$, a valence bond (VB) wave function ${ }^{49,69}$ containing three $\mathrm{VB}$ configurations $\left(\mathrm{A}-\mathrm{B}\right.$, covalent; $\mathrm{A}^{+} \mathrm{B}^{-}$, ionized $\mathrm{A}$; and $\mathrm{A}^{-} \mathrm{B}^{+}$, ionized $\mathrm{B}$ ) is used to describe the electronic structure:

$$
\begin{aligned}
\Psi_{\mathrm{VB}} & =c_{\mathrm{A}^{0} \mathrm{~B}^{0}} \Psi_{\mathrm{A}^{0} \mathrm{~B}^{0}}+c_{\mathrm{A}^{+} \mathrm{B}^{-}} \Psi_{\mathrm{A}^{+} \mathrm{B}^{-}}+\mathrm{c}_{\mathrm{A}^{-} \mathrm{B}^{+}} \Psi_{\mathrm{A}^{-} \mathrm{B}^{+}} \\
& =c_{\mathrm{c}} \Psi_{\mathrm{c}}+c_{\mathrm{I}_{\mathrm{A}}} \Psi_{\mathrm{I}_{\mathrm{A}}}+c_{\mathrm{I}_{\mathrm{B}}} \Psi_{\mathrm{I}_{\mathrm{B}}}
\end{aligned}
$$

For simplicity, we consider only two active electrons; the remaining core electrons are assumed to be localized on the atoms and to shield perfectly, such that the effective nuclear charge on each center, $Z^{\text {eff }}$, may be taken as +1 . Furthermore, we restrict the analysis to the case where the neutral atoms A and $\mathrm{B}$ have a doublet ground state. In the case of the isolated atom $\mathrm{A}$, the ionic species $\mathrm{A}^{+}, \mathrm{A}^{0}$, and $\mathrm{A}^{-}$will be obtained when 0,1 , or 2 valence electrons are placed on it, and analogously for B. The effective Hamiltonian of the system including nuclear repulsion becomes

$$
\hat{H}_{\mathrm{eff}}(1,2 ; R)=\hat{H}(1)+\hat{H}(2)+\frac{1}{r_{12}}+\frac{1}{R}
$$

with

$$
\begin{aligned}
\hat{H}(i) & =\hat{T}(i)+\hat{V}_{\mathrm{A}}(i)+\hat{V}_{\mathrm{B}}(i) \\
& =-\frac{1}{2} \nabla_{i}^{2}-\frac{Z_{\mathrm{A}}^{\mathrm{eff}}}{\left|R_{\mathrm{A}}-\vec{r}_{i}\right|}-\frac{Z_{\mathrm{B}}^{\mathrm{eff}}}{\left|R_{\mathrm{B}}-\vec{r}_{i}\right|}
\end{aligned}
$$

where standard electronic structure theory notation is used. ${ }^{50} \mathrm{~A}$ minimal basis set is adopted, with one normalized, real atomic 
function on each center: $\phi_{\mathrm{A}}(i)$ and $\phi_{\mathrm{B}}(i)$. The normalized wave functions for the VB structures are then

$$
\begin{gathered}
\Psi_{\mathrm{c}}=\Psi_{\mathrm{A}^{0} \mathrm{~B}^{0}}=\frac{1}{\sqrt{2\left(1+S_{\mathrm{AB}}^{2}\right)}}\left(\left|\phi_{\mathrm{A}} \bar{\phi}_{\mathrm{B}}\right\rangle-\left|\bar{\phi}_{\mathrm{A}} \phi_{\mathrm{B}}\right\rangle\right) \\
\Psi_{\mathrm{I}_{\mathrm{A}}}=\Psi_{\mathrm{A}^{+} \mathrm{B}^{-}}=\left|\phi_{\mathrm{B}} \bar{\phi}_{\mathrm{B}}\right\rangle \\
\Psi_{\mathrm{I}_{\mathrm{B}}}=\Psi_{\mathrm{A}^{-} \mathrm{B}^{+}}=\left|\phi_{\mathrm{A}} \bar{\phi}_{\mathrm{A}}\right\rangle
\end{gathered}
$$

where $S_{\mathrm{AB}}(R)=\left\langle\phi_{\mathrm{A}} \mid \phi_{B}\right\rangle$ is an atomic overlap matrix element and the overbar denotes $\beta$ spin. The VB energy $E_{\mathrm{VB}}$ and the normalization condition are

$$
\begin{aligned}
& E_{\mathrm{VB}}=\left\langle\Psi_{\mathrm{VB}}\left|\hat{H}_{\mathrm{eff}}\right| \Psi_{\mathrm{VB}}\right\rangle \\
& =c_{\mathrm{c}}^{2} H_{\mathrm{cc}}+c_{\mathrm{I}_{\mathrm{A}}}^{2} H_{\mathrm{I}_{\mathrm{A}} \mathrm{I}_{\mathrm{A}}}+c_{\mathrm{I}_{\mathrm{B}}}^{2} H_{\mathrm{I}_{\mathrm{B}} \mathrm{I}_{\mathrm{B}}}+2 c_{\mathrm{c}} c_{\mathrm{I}_{\mathrm{A}}} H_{\mathrm{cI}_{\mathrm{A}}}+ \\
& 2 c_{\mathrm{c}} c_{\mathrm{I}_{\mathrm{B}}} H_{\mathrm{cI}_{\mathrm{B}}}+2 c_{\mathrm{I}_{\mathrm{A}}} c_{\mathrm{I}_{\mathrm{B}}} H_{\mathrm{I}_{\mathrm{A}} \mathrm{I}_{\mathrm{B}}} \\
& 1=\left\langle\Psi_{\mathrm{VB}} \mid \Psi_{\mathrm{VB}}\right\rangle \\
& =c_{\mathrm{c}}^{2}+c_{\mathrm{I}_{\mathrm{A}}}^{2}+c_{\mathrm{I}_{\mathrm{B}}}^{2}+2 c_{\mathrm{c}} c_{\mathrm{I}_{\mathrm{A}}} S_{\mathrm{cI}_{\mathrm{A}}}+ \\
& 2 c_{\mathrm{c}} c_{\mathrm{I}_{\mathrm{B}}} S_{\mathrm{cI}_{\mathrm{B}}}+2 c_{\mathrm{I}_{\mathrm{A}}} c_{\mathrm{I}_{\mathrm{B}}} S_{\mathrm{I}_{\mathrm{A}} \mathrm{I}_{\mathrm{B}}}
\end{aligned}
$$

The intrinsically nonorthogonal VB matrix elements can be readily calculated with the Löwdin rules ${ }^{51}$ and Jacobi's ratio theorem. ${ }^{49}$ Defining the atomic terms

$$
\begin{aligned}
E_{\mathrm{A}}^{0} & =h_{\mathrm{AA}} \\
\mathrm{IP}_{\mathrm{A}} & =-h_{\mathrm{AA}} \\
\mathrm{EA}_{\mathrm{A}} & =-h_{\mathrm{AA}}-J_{\mathrm{AA}}
\end{aligned}
$$

and the effective one-electron matrix elements

$$
\begin{aligned}
h_{\mathrm{AB}} & =\left\langle\phi_{\mathrm{A}}\left|\hat{T}+\hat{V}_{\mathrm{A}}+\hat{V}_{\mathrm{B}}\right| \phi_{\mathrm{B}}\right\rangle \\
h_{\mathrm{BC}}^{\mathrm{A}} & =\left\langle\phi_{\mathrm{B}}\left|\hat{T}+\hat{V}_{\mathrm{A}}\right| \phi_{\mathrm{C}}\right\rangle \\
V_{\mathrm{BC}}^{\mathrm{A}} & =\left\langle\phi_{\mathrm{B}}\left|\hat{V}_{\mathrm{A}}\right| \phi_{\mathrm{C}}\right\rangle
\end{aligned}
$$

(where $\phi_{\mathrm{C}}$ is used to denote either of $\phi_{\mathrm{A}}$ or $\phi_{\mathrm{B}}$ ), the VB matrix elements including nuclear repulsion are given as

$$
\begin{gathered}
H_{\mathrm{cc}}=E_{\mathrm{A}^{0} \mathrm{~B} 0}=\frac{1}{\left(1+S_{\mathrm{AB}}^{2}\right)}\left[E_{\mathrm{A}}^{0}+E_{\mathrm{B}}^{0}+F_{\mathrm{c}}(R)\right] \\
H_{\mathrm{I}_{\mathrm{A}} \mathrm{I}_{\mathrm{A}}}=E_{\mathrm{A}^{+} \mathrm{B}^{-}}=E_{\mathrm{A}}^{0}+E_{\mathrm{B}}^{0}+\mathrm{IP}_{\mathrm{A}}-\mathrm{EA}_{\mathrm{B}}+F_{\mathrm{B}}(R) \\
H_{\mathrm{I}_{\mathrm{B}} \mathrm{I}_{\mathrm{B}}}=E_{\mathrm{A}^{-} \mathrm{B}^{+}}=E_{\mathrm{A}}^{0}+E_{\mathrm{B}}^{0}+\mathrm{IP}_{\mathrm{B}}-\mathrm{EA}_{\mathrm{A}}+F_{\mathrm{A}}(R) \\
H_{\mathrm{cI}_{\mathrm{A}}}=\frac{2}{\sqrt{2\left(1+S_{\mathrm{AB}}^{2}\right)}}\left[h_{\mathrm{AB}}+\left(h_{\mathrm{BB}}^{\mathrm{B}}+V_{\mathrm{BB}}^{\mathrm{A}}\right) S_{\mathrm{AB}}+J_{\mathrm{AB}}^{\mathrm{BB}}+\frac{S_{\mathrm{AB}}}{R}\right] \\
H_{\mathrm{cI}_{\mathrm{B}}}=\frac{2}{\sqrt{2\left(1+S_{\mathrm{AB}}^{2}\right)}}\left[h_{\mathrm{AB}}+\left(h_{\mathrm{AA}}^{\mathrm{A}}+V_{\mathrm{AA}}^{\mathrm{B}}\right) S_{\mathrm{AB}}+J_{\mathrm{AB}}^{\mathrm{AA}}+\frac{S_{\mathrm{AB}}}{R}\right] \\
H_{\mathrm{I}_{\mathrm{A}} \mathrm{I}_{\mathrm{B}}}=2 h_{\mathrm{AB}} S_{\mathrm{AB}}+K_{\mathrm{AB}}+\frac{S_{\mathrm{AB}}^{2}}{R}
\end{gathered}
$$

where the Coulomb and exchange integrals are defined as usual and the $F_{i}(R)$ functions are

$$
\begin{aligned}
& F_{\mathrm{c}}(R)=\left[J_{\mathrm{AB}}+K_{\mathrm{AB}}+V_{\mathrm{AA}}^{\mathrm{B}}+V_{\mathrm{BB}}^{\mathrm{A}}+\right. \\
& \left.2 h_{\mathrm{AB}} S_{\mathrm{AB}}+\frac{\left(1+S_{\mathrm{AB}}^{2}\right)}{R}\right] \stackrel{R=\infty}{\longrightarrow} 0 \\
& F_{\mathrm{B}}(R)=\left(2 V_{\mathrm{BB}}^{\mathrm{A}}+\frac{1}{R}\right) \stackrel{R=\infty}{\longrightarrow}-\frac{1}{R} \\
& F_{\mathrm{A}}(R)=\left(2 V_{\mathrm{AA}}^{\mathrm{B}}+\frac{1}{R}\right) \stackrel{R=\infty}{\longrightarrow}-\frac{1}{R}
\end{aligned}
$$

For large $R$, the expected classical electrostatic interactions between the charged species in eq 2.32 are evident. Finally, the VB configuration overlap matrix elements are

$$
\begin{gathered}
S_{\mathrm{cc}}=S_{\mathrm{I}_{\mathrm{A}} \mathrm{I}_{\mathrm{A}}}=S_{\mathrm{I}_{\mathrm{B}} \mathrm{I}_{\mathrm{B}}}=1 \\
S_{\mathrm{cI}_{\mathrm{A}}}=S_{\mathrm{cI}_{\mathrm{B}}}=\frac{2 S_{\mathrm{AB}}}{\sqrt{2\left(1+S_{\mathrm{AB}}^{2}\right)}} \\
S_{\mathrm{I}_{\mathrm{A}} \mathrm{I}_{\mathrm{B}}}=S_{\mathrm{AB}}^{2}
\end{gathered}
$$

The definition of atomic partial charge given a molecular wave function is a critical point in our discussion. This topic and the closely related question of how atoms can be defined inside molecules have a long history in quantum chemistry. ${ }^{52} \mathrm{We}$ mention only the approaches closely related to Mulliken analysis: Mulliken, ${ }^{53,54}$ Coulson, ${ }^{55}$ and Löwdin ${ }^{56}$ charges. All three of these definitions are equivalent within the zero differential overlap (ZDO) approximation. ${ }^{57,58}$ The reader is referred to the original references for the detailed definitions of these charges; we only quote the result for the Coulson charges as they appear in the specific VB model we have described:

$$
\begin{array}{rr}
N_{\mathrm{A}}^{(\text {Coul })}=c_{\mathrm{c}}^{2}+2 c_{\mathrm{I}_{\mathrm{B}}}^{2} & q_{\mathrm{A}}^{(\text {Coul })}=1-c_{\mathrm{c}}^{2}-2 c_{\mathrm{I}_{\mathrm{B}}}^{2} \\
N_{\mathrm{B}}^{(\text {Coul })}=c_{\mathrm{c}}^{2}+2 c_{\mathrm{I}_{\mathrm{A}}}^{2} & q_{\mathrm{B}}^{(\text {Coul })}=1-c_{\mathrm{c}}^{2}-2 c_{\mathrm{I}_{\mathrm{A}}^{2}}^{2} \\
N_{\mathrm{A}}^{(\text {Coul })}+N_{\mathrm{B}}^{(\text {Coul })}=2 & q_{\mathrm{A}}^{(\text {Coul })}=-q_{\mathrm{B}}^{(\text {Coul })}=q^{(\text {Coul })}
\end{array}
$$

Given a convenient VB model for the diatomic molecule $A B$, we now consider the relationship between the GC and VB theories. An immediate difficulty is that the VB energy will be a function of three coefficients (given the normalization constraint only two are independent), but the GC and CE theories have only one parameter for this case: the charge transfer $q$. Thus, we need to introduce a mapping between VB wave functions and $q$. Given the set of all wave functions that are consistent with the desired charge, there are two natural ways to proceed. The first is to choose the wave function that provides the minimal energy within the subspace (a constrained search procedure as has been used in a different context in DFT).$^{59}$ The second is to invoke the ideas of $\mathrm{ME},{ }^{60-62}$ defining the energy as an equally weighted average over the energies of all wave functions consistent with the constraints. The optimal values of the constraints are then determined by energy minimization, as discussed in the Introduction. It is this second approach that is most fruitful in relating to GC and CE theories. This approach is partially justified by the result: we will see below that the GC and CE models are particular cases of this 
maximum entropy VB (MEVB) model. However, as discussed in the Introduction, it is also justified as the best physical solution consistent with a description where only the constraint variables (in this case the atomic partial charges) are considered "knowable."

The energy $E_{\mathrm{AB}}^{\mathrm{VB}}$ of an arbitrary $\mathrm{VB}$ wave function can be split into the diagonal and off-diagonal parts:

$$
\begin{aligned}
& E_{\mathrm{AB}}^{\mathrm{VB}}\left(c_{\mathrm{c}}, c_{\mathrm{I}_{\mathrm{A}}}, c_{\mathrm{I}_{\mathrm{B}}}\right)=E_{\mathrm{AB}}^{\mathrm{VB} \text { on }}(\vec{c})+E_{\mathrm{AB}}^{\mathrm{VB} \text { off }}(\vec{c}) \\
& E_{\mathrm{AB}}^{\mathrm{VB} \text { on }}(\vec{c})=c_{\mathrm{c}}^{2} H_{\mathrm{cc}}+c_{\mathrm{I}_{\mathrm{A}}}^{2} H_{\mathrm{I}_{\mathrm{A}} \mathrm{I}_{\mathrm{A}}}+c_{\mathrm{I}_{\mathrm{B}}}^{2} H_{\mathrm{I}_{\mathrm{B}} \mathrm{I}_{\mathrm{B}}} \\
& E_{\mathrm{AB}}^{\mathrm{VB} \text { off }}(\vec{c})=2 c_{\mathrm{c}} c_{\mathrm{I}_{\mathrm{A}}} H_{\mathrm{cI}_{\mathrm{A}}}+2 c_{\mathrm{c}} c_{\mathrm{I}_{\mathrm{B}}} H_{\mathrm{cI}_{\mathrm{B}}}+2 c_{\mathrm{I}_{\mathrm{A}}} c_{\mathrm{I}_{\mathrm{B}}} H_{\mathrm{I}_{\mathrm{A}} I_{\mathrm{B}}}
\end{aligned}
$$

The diagonal part, $E_{\mathrm{AB}}^{\mathrm{VB} \text { on }}$ has the same form as $E_{\mathrm{AB}}^{\mathrm{GC} \beta \rightarrow \infty}(q)$, suggesting that we choose the following relations to define the mapping between charge and coefficients:

$$
\begin{aligned}
c_{\mathrm{c}}^{2} & =[1+q-2 q \Theta(q)] \\
c_{\mathrm{I}_{\mathrm{A}}}^{2} & =[q \Theta(q)] \\
c_{\mathrm{I}_{\mathrm{B}}}^{2} & =q[\Theta(q)-1]
\end{aligned}
$$

For a given value of $q$ on the allowed domain, there are eight vectors $\left(c_{\mathrm{c}}, c_{\mathrm{I}_{\mathrm{A}}}, c_{\mathrm{I}_{\mathrm{B}}}\right)_{i}$ consistent with this map, having $c_{\mathrm{c}}=$ $\pm \sqrt{1+q-2 q \Theta(q)}, c_{\mathrm{I}_{\mathrm{A}}}= \pm \sqrt{q \Theta(q)}$, and $c_{\mathrm{I}_{\mathrm{B}}}= \pm \sqrt{q[\Theta(q)-1]}$. Averaging the eight resulting $\mathrm{VB}$ energies $E_{\mathrm{AB}}^{\mathrm{VB}}\left[\left(c_{\mathrm{c}}, c_{\mathrm{I}_{\mathrm{A}}}, c_{\mathrm{I}_{\mathrm{B}}}\right)_{i}\right]$ leads to

$$
E_{\mathrm{AB}}^{\mathrm{VB} \text { Average } \mathrm{GC}}=\frac{1}{8} \sum_{i=1}^{8} E_{\mathrm{AB}}^{\mathrm{VB}}\left[\left(c_{\mathrm{c}}, c_{\mathrm{I}_{\mathrm{A}}}, c_{\mathrm{I}_{\mathrm{B}}}\right)_{i}\right]=E_{\mathrm{AB}}^{\mathrm{VB} \text { on }}(q)
$$

where the off-diagonal terms in the energy have canceled. At infinite separation, it automatically holds that $E_{\mathrm{AB}}^{\mathrm{VB}}=E_{\mathrm{AB}}^{\mathrm{VB} \text { on }}$; however, the above mapping produces a diagonal energy expression for all bond distances. Therefore,

$$
\begin{aligned}
E_{\mathrm{AB}}^{\mathrm{VB} \text { Average } \mathrm{GC}}(q, R)= & E_{\mathrm{A}^{0} \mathrm{~B}^{0}}(R)[1+q-2 q \Theta(q)]+ \\
& E_{\mathrm{A}^{+} \mathrm{B}^{-}}(R)[q \Theta(q)]+ \\
& E_{\mathrm{A}^{-} \mathrm{B}^{+}}(R) q[\Theta(q)-1]
\end{aligned}
$$

where the dependence on the bond distance $R$ has been made explicit. Notice that $E_{\mathrm{AB}}^{\mathrm{VB} \text { Average } \mathrm{GC}}$ can be viewed as a generalization of $E_{\mathrm{AB}}^{\mathrm{GC} \beta \rightarrow \infty}$ for all bond distances satisfying $E_{\mathrm{AB}}^{\mathrm{VB} \text { Average } \mathrm{GC}}=E_{\mathrm{AB}}^{\mathrm{GC} \beta \rightarrow \infty}$ at $R=\infty$.

The minimum of $E_{\mathrm{AB}}^{\mathrm{VB} \text { Average } \mathrm{GC}}(q, R)$ will not necessarily stay at the point $q_{\min }=0$. Since the derivative $\partial E_{\mathrm{AB}}^{\mathrm{VB} \text { Average } \mathrm{GC}}(q ; R) / \partial q$ does not exist for integer $q$, it is impossible to find the minimum by setting $\partial E_{\mathrm{AB}}^{\mathrm{VB}}$ Average $\mathrm{GC}_{\left(q_{\min }, R\right) / \partial q}$ $=0$. Instead, a direct value inspection must be done in order to keep track of the minimum as $R$ varies. This can be done by a quasi-analytical procedure if the integrals occurring in the terms

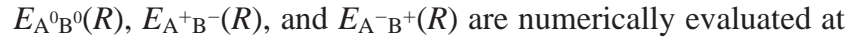
different bond separations and the final energy is calculated for each. However, a useful analytical procedure can be devised if some approximations are introduced. Approximating the interaction functions $F_{\mathrm{c}}(R), F_{\mathrm{A}}(R)$, and $F_{\mathrm{B}}(R)$ by their asymptotic values, the energies in the weighted sum become

$$
\begin{aligned}
& E_{\mathrm{A}^{0} \mathrm{~B}^{0}}(R) \approx E_{\mathrm{A}}^{0}+E_{\mathrm{B}}^{0} \\
& E_{\mathrm{A}^{+} \mathrm{B}^{-}}(R) \approx E_{\mathrm{A}}^{+}+E_{\mathrm{B}}^{-}-\frac{1}{R} \\
& E_{\mathrm{A}^{-} \mathrm{B}^{+}}(R) \approx E_{\mathrm{A}}^{-}+E_{\mathrm{B}}^{+}-\frac{1}{R}
\end{aligned}
$$

These expressions are completely classical in nature and the energy $E_{\mathrm{A}^{0} \mathrm{~B}^{0}}$ becomes independent of $R$. At some critical distance $R_{\text {crit }}$, the energy of one of the ionic terms will fall below the covalent energy, and $q_{\min }$ will switch abruptly from 0 to \pm 1 . The piecewise-linear behavior of $E_{\mathrm{AB}}^{\mathrm{VB} \text { Average } \mathrm{GC}}(q ; R)$ will remain for all $R$. The exact quasi-analytical procedure mentioned above will render exactly the same result of abrupt charge transfer at $R_{\text {crit }}$ although the value of $R_{\text {crit }}$ will be different.

To relate the $\mathrm{VB}$ and $\mathrm{CE}$ theories, we again appeal to $\mathrm{ME}$ ideas, adopting a mapping between $\mathrm{VB}$ wave functions and $\mathrm{CE}$ theory based on the Coulson charge. The Coulson charge definition implies a zero differential overlap (ZDO) model, ${ }^{57,58}$ so it is appropriate to use a normalization expression that approximates $S_{\mathrm{AB}}=0$. Using this normalization constraint and demanding that eq 2.35 hold, we find $c_{\mathrm{c}}^{2}=\left(1-q^{2}\right), c_{\mathrm{I}_{\mathrm{A}}}{ }^{2}=$ $1 / 2\left(q^{2}+q\right)$, and $c_{\mathrm{I}_{\mathrm{B}}}{ }^{2}=1 / 2\left(q^{2}-q\right)$. There are again eight vectors $\left(c_{1}, c_{2}, c_{3}\right)$ satisfying the conditions with $c_{\mathrm{c}}= \pm \sqrt{1-q^{2}}$, to $c_{\mathrm{I}_{\mathrm{A}}}= \pm \sqrt{1 / 2\left(q^{2}+q\right)}$, and $c_{\mathrm{I}_{\mathrm{B}}}= \pm \sqrt{1 / 2\left(q^{2}-q\right)}$. Although $c_{\mathrm{c}}$ is always real for $q$ in the allowed domain, one of $c_{\mathrm{I}_{\mathrm{A}}}$ and $c_{\mathrm{I}_{\mathrm{B}}}$ is imaginary for noninteger $q$. Averaging over the energies of these eight wave functions, one finds

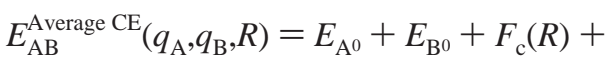

$$
\begin{aligned}
& {\left[\chi_{\mathrm{A}}^{0}-\frac{1}{2} F_{\mathrm{A}}(R)\right] q_{\mathrm{A}}+} \\
& {\left[\chi_{\mathrm{B}}^{0}-\frac{1}{2} F_{\mathrm{B}}(R)\right] q_{\mathrm{B}}+} \\
& \eta_{\mathrm{A}}^{0} q_{\mathrm{A}}^{2}+\eta_{\mathrm{B}}^{0} q_{\mathrm{B}}^{2}+ \\
& \frac{1}{2} q_{\mathrm{A}} q_{\mathrm{B}}\left[2 F_{\mathrm{c}}(R)-F_{\mathrm{A}}(R)-F_{\mathrm{B}}(R)\right]
\end{aligned}
$$

where the off-diagonal terms have again canceled out. At infinite separation, this equation reduces to the CE energy expression. However, there are a few points to be made for finite bond distances. First, the presence of $F_{\mathrm{c}}(R)$ in the first line of eq 2.41 reflects the covalent part of the A-B interaction. Since it represents a $q$-independent shift of the energy, it will have no influence on the optimal charges as determined by minimization of eq 2.41 at fixed $R$. Second, the electronegativity is now environment-dependent:

$$
\chi_{\mathrm{A}}(R)=\chi_{\mathrm{A}}^{0}-\frac{1}{2} F_{\mathrm{A}}(R)
$$

with an analogous expression for $\chi_{\mathrm{B}}(R)$. Itskowitz and Berkowitz have arrived at a similar form for an environment-dependent electronegativity using a quite different method based on density functional theory. ${ }^{63}$ The $\mathrm{CE}$ model does not contain this environment dependence, which can be interpreted to mean that an average or representative value of the atomic electronegativity is implicitly used, e.g., $\chi_{\mathrm{A}}(R) \approx \chi_{\mathrm{A}}\left(R_{\text {eq }}\right)$. Finally, if we apply Mulliken-Ruedenberg approximations ${ }^{64,65}$ to the integrals in $F_{\mathrm{c}}(R)$, consistent with the ZDO origin of the Coulson charge, and neglect all terms higher than first-order in $S_{\mathrm{AB}}$, we find 


$$
\frac{1}{2}\left[2 F_{\mathrm{c}}(R)-F_{\mathrm{A}}(R)-F_{\mathrm{B}}(R)\right] \rightarrow J_{\mathrm{AB}}
$$

We conclude that the CE expression is exactly recovered (with a shielded Coulomb interaction as employed by Rappe and Goddard $^{27}$ and an environment-dependent electronegativity) from the application of a charge-constrained maximum entropy procedure to a minimal basis VB wave function given the ZDO approximation and the Coulson definition of atomic charge.

It is fruitful to compare these results to previous work in the context of DFT. For the case of a neutral diatomic AB, the CE charge $q_{\mathrm{A}}=-q_{\mathrm{B}}$ is simply

$$
q_{\mathrm{A}}=\frac{\left(\chi_{\mathrm{B}}^{0}-\chi_{\mathrm{A}}^{0}\right)}{2\left[\eta_{\mathrm{A}}^{0}+\eta_{\mathrm{B}}^{0}-J_{\mathrm{AB}}\left(R_{\mathrm{AB}}\right)\right]}
$$

The MEVB-CE charge is given by

$$
q_{\mathrm{A}}=\frac{\left(\chi_{\mathrm{B}}^{0}-\chi_{\mathrm{A}}^{0}\right)+\frac{1}{2} F_{\mathrm{A}}\left(R_{\mathrm{AB}}\right)-\frac{1}{2} F_{\mathrm{B}}\left(R_{\mathrm{AB}}\right)}{2\left[\eta_{\mathrm{A}}^{0}+\eta_{\mathrm{B}}^{0}-J_{\mathrm{AB}}\left(R_{\mathrm{AB}}\right)\right]}
$$

differing only in the environment dependence of the electronegativities. By combining the Sanderson Principle with the DFT expression for the chemical potentials $\mu_{A}$ and $\mu_{B}$ as a function of the atomic charges, Nalewajski ${ }^{66}$ has derived

$$
q_{\mathrm{A}}=\frac{\left(\chi_{\mathrm{B}}^{0}-\chi_{\mathrm{A}}^{0}\right)+\int f_{\mathrm{A}}(R) \Delta v_{\mathrm{A}}(R) \mathrm{d} R-\int f_{\mathrm{B}}(R) \Delta v_{\mathrm{B}}(R) \mathrm{d} R}{2\left[\eta_{\mathrm{A}}^{0}+\eta_{\mathrm{B}}^{0}\right]}
$$

where $f_{\mathrm{A}}$ and $f_{\mathrm{B}}$ are the Fukui functions ${ }^{67,68}$ of atoms $\mathrm{A}$ and $\mathrm{B}$, and $\Delta v_{\mathrm{A}}$ and $\Delta v_{\mathrm{B}}$ are the external potential perturbation on atoms $A$ and $B$ by their counterparts $\mathrm{B}$ and $\mathrm{A}$, respectively, In a more sophisticated perturbation-DFT approach, Itskowitz and Berkowitz $^{63}$ derived

$$
q_{\mathrm{A}}=\frac{\left(\chi_{\mathrm{B}}^{0}-\chi_{\mathrm{A}}^{0}\right)+\int f_{\mathrm{A}}(R) \Delta v_{\mathrm{A}}(R) \mathrm{d} R-\int f_{\mathrm{B}}(R) \Delta v_{\mathrm{B}}(R) \mathrm{d} R}{2\left[\eta_{\mathrm{A}}^{0}+\eta_{\mathrm{B}}^{0}-J_{\mathrm{AB}}\left(R_{\mathrm{AB}}\right)\right]}
$$

This equation has the same form as eq 2.45, and becomes identical to it under the approximation that $f_{\mathrm{A}} \approx \phi_{\mathrm{A}}{ }^{2}$ and $f_{\mathrm{B}} \approx$ $\phi_{\mathrm{B}}{ }^{2}$. The environment dependence of the electronegativities cannot repair the dissociation problem. By imposing the condition of chemical equilibrium $\mu_{\mathrm{A}}=\mu_{\mathrm{B}}$ at all bond separations, Berkowitz ${ }^{47}$ has derived

$$
q_{\mathrm{A}}=\frac{\int f_{\mathrm{A}}(R) \Delta v_{\mathrm{A}}(R) \mathrm{d} R-\int f_{\mathrm{B}}(R) \Delta v_{\mathrm{B}}(R) \mathrm{d} R}{2\left[\eta_{\mathrm{A}}^{0}+\eta_{\mathrm{B}}^{0}-J_{\mathrm{AB}}\left(R_{\mathrm{AB}}\right)\right]}
$$

which does correctly give a charge $q_{\mathrm{A}}=0$ for the dissociation in the gas phase. However, the conspicuous absence of the electronegativity term $\left(\chi_{\mathrm{B}}^{0}-\chi_{\mathrm{A}}^{0}\right)$ will make this formula qualitatively unrealistic for near-equilibrium geometries. A globally accurate CE expression does not yet exist in any DFTbased theory.

\section{Conclusions}

We have explored the origin of and relationships between various classical charge transfer theories. A purely statistical approach (GC) that has been widely studied by previous workers has been shown to give rise to the charge equilibration (CE) theory for a special case where the temperature is complex. We have introduced a maximum entropy-motivated mapping between atomic charges and the underlying wave function(s): maximum entropy valence bond (MEVB) theory.

We have shown that both the GC and CE approaches can be considered as specialized cases of MEVB, with different definitions for the charge that enters as a constraint. To the best of our knowledge, this work represents the first unification of GC and CE approaches. More importantly, our approach suggests many other possible models that have yet to be explored. Entire families of classical charge transfer methods can be generated by applying these procedures with other definitions of atomic partial charges, for example, Mulliken or Löwdin charges. Additionally, one can proceed beyond the minimal basis VB wave function used here. The expansion of the number of degrees of freedom implicit in a more flexible VB wave function should be accompanied by an extension of the constraints from charges to include also higher multipoles and response functions, e.g., dipoles and polarizabilities. These are very promising new areas of research that will hopefully culminate both in the improvement of classical charge transfer/ polarizability models and also in a rigorous and well-defined framework for interfacing classical electrostatic models with quantum chemistry. We have not needed to use the formal tools of maximum entropy methods in this work because of the simplicity of the model. However, this situation will change for the more complicated extensions we mention.

Our aim in this paper has been primarily conceptual: to introduce a new, rigorously derived way of formulating the problem of quantum chemistry in open systems that emphasizes wave functions instead of densities. However, one should realize that the MEVB model inherits the problems of GC and $\mathrm{CE}$ theories insofar as it reduces to them. The practical problem is to remove the limitations of these theories. Because the MEVB theory is derived in a well-defined way from wave functions, one can identify precisely the steps that lead to breakdown, for example the residual charge transfer in the CE representation of infinitely separated atoms. Indeed, we have already formulated an MEVB-based model that removes this problem while retaining most of the simplicity of the CE theory. ${ }^{70}$ This work will be presented in the sequel to this paper.

Acknowledgment. We are pleased to dedicate this paper to Bill Miller on the occasion of his 60th birthday, whose attempts to understand electrons in a classical framework have inspired us. We thank Profs. P. G. Wolynes and R. D. Levine for their insightful comments. This work was supported by the National Science Foundation (CHE-97-33403 and DMR-99-76550) and the Beckman Foundation. T.J.M. is grateful to the NSF, Beckman Foundation, Packard Foundation, Sloan Foundation, Dreyfus Foundation, and Research Corp. for CAREER, Beckman Young Investigator, Packard Fellow, Sloan Fellow, Dreyfus Teacher-Scholar, and Research Innovation awards, respectively.

\section{References and Notes}

(1) Nakatsuji, H. J. Chem. Phys. 1987, 87, 4995.

(2) Artacho, E.; Falicov, L. M. Phys. Rev. B 1993, 47, 1190.

(3) Goddard, W. A.; McGill, T. C. J. Vac. Sci. Technol. 1979, 16, 1308. 2607.

(4) McAdon, M. H.; Goddard, W. A., III. J. Phys. Chem. 1987, 91,

(5) Wu, C. J.; Carter, E. A. J. Am. Chem. Soc. 1991, 113, 9061.

(6) Siegbahn, P. E. M. Surf. Sci. 1992, 269, 276.

(7) Blomberg, M. R. A.; Siegbahn, P. E. M. Mol. Phys. 1999, 96, 571.

(8) Moore, D. B.; Martínez, T. J. J. Phys. Chem. A 2000, 104, 2367. 

1968.

(9) Benson, S. Thermonuclear Kinetics; Wiley and Sons: New York,

(10) Tomasi, J.; Bonaccorsi, R.; Cammi, R.; Olivares del Valle, F. J. J. Mol. Struct. (THEOCHEM) 1991, 234, 401.

(11) Tannor, D. J.; Marten, B.; Murphy, R.; Friesner, R. A.; Sitkoff, D.; Nicholls, A.; Ringnalda, M.; Goddard, W. A., III; Honig, B. J. Am. Chem. Soc. 1994, 116, 11875.

(12) Warshel, A. Computer Modeling of Chemical Processes in Enzymes and Solutions; Wiley: New York, 1991.

(13) Day, P. N.; Jensen, J. H.; Gordon, M. S.; Webb, S. P.; Stevens, W. J.; Krauss, M.; Garmer, D.; Basch, H.; Cohen, D. J. Chem. Phys. 1996, 105,1968

(14) Warshel, A.; Levitt, M. J. Mol. Biol. 1976, 103, 227.

(15) Field, M. J.; Bash, P. A.; Karplus, M. J. Comput. Chem. 1990, 11, 700 .

(16) In this paper, we use the words quantum and classical in reference to the electronic structure problem, implying that a wave function is or is not the primary representation, respectively. This should not be confused with the related, but quite different, problem of coupling classical (or semiclassical) and quantum mechanical representations of the nuclear degrees of freedom.

(17) Chiles, R. A.; Jongeward, G. A.; Bolton, M. A.; Wolynes, P. G. J. Chem. Phys. 1984, 81, 2039.

(18) Sanderson, R. T. Science 1951, 144, 670.

(19) Pauling, L.; Yost, D. M. Proc. Natl. Acad. Sci. U.S.A. 1932, 14 414.

(20) Iczokowski, R. P.; Margrave, J. L. J. Chem. Soc. 1961, 83, 3547.

(21) Hinze, J.; Jaffe, H. H. J. Chem. Phys. 1962, 84, 540.

(22) Janak, J. F. Phys. Rev. B 1978, 18, 7165.

(23) Parr, R. G.; Donnelly, R. A.; Levy, M.; Palke, W. E. J. Chem. Phys. 1978, 68, 3801.

(24) Mulliken, R. S. J. Chem. Phys. 1934, 2, 782.

(25) Gasteiger, J.; Marsili, M. Tetrahedron 1980, 36, 3219.

(26) Del Re, G.; Pullman, B.; Yonezawa, T. Biochim. Biophys. Acta 1963, 75, 153 .

(27) Rappe, A. K.; Goddard, W. A., III. J. Phys. Chem. 1991, 95, 3358 6141 .

(28) Rick, S. W.; Stuart, S. J.; Berne, B. J. J. Chem. Phys. 1994, 101 ,

(29) Banks, J. L.; Kaminski, G. A.; Zhou, R.; Mainz, D. T.; Berne, B. J.; Friesner, R. A. J. Chem. Phys. 1999, 110, 741.

(30) York, D. M.; Yang, W. J. Chem. Phys. 1996, 104, 159.

(31) Chelli, R.; Procacci, P.; Righini, R.; Califano, S. J. Chem. Phys. 1999, $111,8569$.

(32) Ben-Nun, M.; Martinez, T. J. Chem. Phys. Lett. 1998, 290, 289.

(33) Assfeld, X.; Rivail, J. L. Chem. Phys. Lett. 1996, 263, 100.

(34) Murphy, R. B.; Philipp, D. M.; Friesner, R. A. Chem. Phys. Lett. 2000, 321, 113

(35) Kairys, V.; Jensen, J. H. J. Phys. Chem. A 2000, 104, 6656.

(36) Levine, R. D. Annu. Rev. Phys. Chem. 1978, 29, 59.

(37) Tishby, N. Z.; Levine, R. D. Chem. Phys. Lett. 1984, 104, 4.

(38) Parr, R. G.; Person, R. G. J. Am. Chem. Soc. 1983, 105, 1503.
(39) Parr, R. G.; Yang, W. Density-Functional Theory of Atoms and Molecules; Oxford University Press: Oxford, U.K., 1989.

(40) Perdew, J. P.; Parr, R. G.; Levy, M.; Balduz, J. L. Phys. Rev. Lett. 1982, 49, 1691.

(41) Gyftopoulos, E. P.; Hatsopoulos, G. N. Proc. Natl. Acad. Sci. U.S.A 1968, 60, 786

(42) Abdulnur, S. F.; Linderberg, J.; Ohrn, U.; Thulstrup, P. W. Phys. Rev. A 1972, 6, 889 .

(43) Slater, J. C.; Mann, J. B.; Wilson, T. M.; Wood, J. H. Phys. Rev. 1969, 184, 672 .

(44) Arfken, A. Mathematical Methods for Physicists; Academic Press Inc.: Boston, 1985

(45) Tolman, R. C. The Principles of Statistical Mechanics; Dover Publication, Inc.: New York, 1979.

(46) It would be quite reasonable to consider the system existing within a cavity of fixed size in the bath. In this case, there will be kinetic energy and Coulomb repulsion terms disfavoring the addition of electrons, ad infinitum to the system.

(47) Berkowitz, M. J. Am. Chem. Soc. 1987, 109, 4823.

(48) Rychlewski, J.; Parr, R. G. J. Chem. Phys. 1986, 84.

(49) McWeeney Methods of Molecular Quantum Mechanics; Academic

Press: San Diego, CA, 1992

(50) Szabo, A.; Ostlund, N. S. Modern Quantum Chemistry; Dover Publications: Mineola, New York, 1989.

(51) Löwdin, P. O. Phys. Rev. 1955, 97, 1474

(52) Bader, R. F. W. Atoms in Molecules: A Quantum Theory; Clarendon Press: Oxford, U.K., 1995.

(53) Mulliken, R. S. J. Chem. Phys. 1955, 23, 1833.

(54) Mulliken, R. S. J. Chem. Phys. 1955, 23, 2343

(55) Li, J.; Williams, B.; Cramer, J. C.; Thrular, D. G. J. Chem. Phys. 1999, 110,724

(56) Lowdin, P. O. J. Chem. Phys. 1950, 18, 365.

(57) Pople, J. A.; Santry, D. P.; Segal, G. A. J. Chem. Phys. 1965, 43, S129.

(58) Pople, J. A.; Segal, G. A. J. Chem. Phys. 1965, 43, S136.

(59) Levy, M. Proc. Natl. Acad. Sci. U.S.A. 1979, 76, 6062

(60) Levine, R. D. J. Phys. A 1980, 13, 91

(61) Levine, R. D.; Tribus, M. The Maximum Entropy Formalism; MIT Press: Cambridge, MA, 1978.

(62) Jaynes, E. T. Phys. Rev. 1957, 106, 620.

(63) Itskowitz, P.; Berkowitz, M. J. Phys. Chem. A 1997, 101, 5687.

(64) Mulliken, R. S. J. Chim. Phys. 1949, 46, 500.

(65) Ruedenberg, K. J. Chem. Phys. 1951, 19, 1433

(66) Nalewajski, R. F. J. Am. Chem. Soc. 1984, 106, 944

(67) Fukui, K. Science 1987, 218, 747.

(68) Parr, R. G.; Yang, W. J. Am. Chem. Soc. 1984, 106, 4049.

(69) Bobrowicz, F. W.; Goddard, W. A., III. Self-Consistent Field Equations for the Generalized Valence Bond and Open-Shell Hartree-Fock Wave Functions. In Methods of Electronic Structure Theory; Schaefer, H. F., III, Ed; Plenum Press: New York and London, 1977; Vol. 3.

(70) Morales, J. A.; Martinez, T. J. Manuscript in preparation, 2001. 